\title{
Evaluación de un método de intervención del clima organizacional (CO) en una empresa de servicios públicos colombiana
}

\author{
Lina Marcela Guevara Bedoya ${ }^{1}$ \& Maria Eugenia Londoño Londoño ${ }^{2}$ \\ Recibido: 25/10/2017 Aceptado: 18/06/2018 \\ DOI: $10.21772 /$ ripo.v36n2a04
}

\begin{abstract}
Resumen
En esta investigación se presentan los efectos de la implementación de una metodología para la gestión del Clima Organizacional (CO) en una organización de servicios públicos colombiana. Se realizó un pretest con 157 personas y, con base en los resultados, se implementaron talleres para los líderes y se construyeron planes de acción con los equipos. Para la formulación de estos planes se realizó una búsqueda de explicaciones con la técnica de grupos nominales y la formulación de estrategias de acción con el grupo. Tres meses después se hizo seguimiento y dos años más tarde un postest con 169 colaboradores y se compararon los resultados de cada variable. De acuerdo con la distribución de los datos se utilizaron pruebas no paramétricas para la comparación de las puntuaciones. Adicionalmente se calculó el tamaño del efecto. Se encontraron mejoras estadísticamente significativas y con un efecto grande en todos los atributos a excepción de Retribución. De los anteriores resultados se concluye que se cuenta con una metodología válida para la intervención del CO.
\end{abstract}

Palabras clave: clima organizacional, intervención.

\section{Evaluation of a Methodology for the Management of the Organizational Climate (OC) in a Colombian Utilities Organization}

\begin{abstract}
This research presents the effects of the implementation of a methodology for the management of the Organizational Climate (OC) in a Colombian utilities organization. A pre-test was conducted with 157 people and, based on the results, workshops were held for the leaders and action plans were built with the teams. For the formulation of these plans, a search fo explanatios was made with the nominal group technique and the formulation of action strategies with the group. Three months later we monitored the process, two years later a post-test with 169 collaborators was conducted and the results of each variable were compared. According to the data distribution, non-parametric tests were used for the comparison of the scores. Additionally, the size of the effect was calculated. Statistically significant improvements were found with a large effect on all attributes except for Retribution. From the above results it is concluded that there is a valid methodology for OC intervention
\end{abstract}

Keywords: Organizational Climate, Intervention.

1 Psicóloga. Magister en Desarrollo Humano Organizacional de la Universidad EAFIT. Especialista en Gerencia del Desarrollo Humano. Centro de Investigación en Comportamiento Organizacional Medellín-Colombia. E-mail: lguevara@cincel.com.co

2 Psicóloga. Especialista en Psicología Organizacional y Salud Ocupacional de la Universidad de Antioquia. Magister en Psicología de las Organizaciones, del Trabajo y de los Recursos Humanos de la Universidad Jaume I de España. Lidera el área de Diseño de Sistemas e Instrumentos Evaluativos en Cincel. E-mail: mariaeugenial@cincel.com.co

Nota: El presente artículo fue publicado en el 2018. Sin embargo, con el fin de mantener la periodicidad de la Revista, el número corresponde al segundo semestre del año 2017.

Cómo citar este artículo: Guevara Bedoya, L. \& Londoño Londoño, M. E. (2017). Evaluación de un Método de Intervención del Clima Organizacional (CO) en una Empresa de Servicios Públicos Colombiana. Revista Interamericana de Psicología Ocupacional, 36(2), 46-57. DOI: 10.21772/ripo. v36n2a04 


\section{Introducción}

En los últimos años se ha presentado un llamativo incremento en la atención que las organizaciones le prestan al tema del Clima Organizacional (CO) (Vega, Arévalo, Sandoval, Aguilar-Bustamante \& Giraldo, 2006) dado que ha demostrado tener un impacto en fenómenos empresariales tales como la rotación, la satisfacción laboral, el desempeño en el trabajo, la satisfacción del cliente, la calidad del servicio y los resultados financieros (González-Roma, Peiro y Tordera, 2002; Ostroff, Kinicki, \& Tamkins, 2003).

Otro aspecto que ha incrementado la atención hacia el tema son los requerimientos que se realizan en los procesos de acreditación en normas de calidad (Vega et al., 2006). Si bien es muy común encontrar este interés por evaluar o describir la calidad del $\mathrm{CO}$ en las organizaciones, específicamente en las colombianas (Vega et al., 2006) y la realización de acciones que buscan el mejoramiento de los resultados, al indagar sobre el tema se encuentra escasa información sobre la efectividad de las intervenciones que se realizan (Nielsen, Taris \& Cox, 2010). La mayoría de estas experiencias se encuentra en la denominada literatura gris, es decir en medios que no están publicados en canales que permitan el acceso a la información (OMS, 2010). Por esta razón, el presente artículo busca presentar los efectos de una metodología para la gestión del $\mathrm{CO}$ que demostró efectividad en una organización de servicios públicos colombiana.

El concepto de Clima Organizacional (CO) se ha trabajado a partir de la década de 1960 derivado de investigaciones de psicología social de autores como Lewin (1951) y Lewin, Lippitt \&White (1939) (Mahal, 2009) quienes argumentaron que el comportamiento de las personas en el trabajo no depende únicamente de las características personales de los trabajadores, sino de la interacción de estas con las condiciones organizacionales. Algunos autores plantean que el CO y la cultura son definiciones sinónimas (Mahal, 2009), no obstante, otros han hecho una clara diferenciación de estos dos fenómenos planteando que el $\mathrm{CO}$ es uno de los componentes de la cultura organizacional (Toro \& Cabrera, 1998). Si bien es común encontrar múltiples definiciones de este concepto (Argyris, 1957; Brunet, 1987; Cardenas, Arciniegas, Barrera, 2009; Chiavenato, 1992; Davis, 1991; Mahal, 2009; Méndez Álvarez, 2006; Ostroff, Kinicki, \& Tamkins, 2003) estas coinciden en concebir este fenómeno como un proceso perceptivo en relación con realidades específicas del trabajo.

Paraefectosdelpresenteartículosetrabajará con la definición clásica de Reichers y Schneider (1990), que ha sido bastante retomada en estudios con empresas colombianas (Vega, et al., 2006). Estos autores se refieren a este fenómeno como las imágenes, representaciones o percepciones colectivas que elaboran los miembros de un equipo o de una organización en relación con las distintas realidades de su trabajo. Esta definición, al hacer alusión al fenómeno perceptivo, implica que puede corresponder o no a la realidad, pero que tendrá un impacto en los juicios y reacciones de las personas ante sus realidades laborales y, por ende, en el comportamiento que exhiben. Esta característica también evidencia que es un constructo psicosocial en tanto que surge de la interacción de las condiciones individuales con el contexto del trabajo. Al mencionar el término compartidas, se hace alusión a que generalmente se obtienen los puntos de vista individuales de los miembros de las empresas y luego estos se agregan para conocer el $\mathrm{CO}$ de la misma. Algunos autores plantean que debe existir un alto nivel de acuerdo en la manera como las personas perciben las condiciones para que se pueda hablar de clima organizacional (Gonzalez-Roma, Peiró, \& Tordera, 2002), no obstante, otra visión plantea que este es un aspecto configuracional de los grupos de trabajo y que, por ende, se puede hablar de clima organizacional aun en ausencia de consenso, dado que esta alta dispersión constituirá 
precisamente la caracterización del mismo. Por último, la definición planteada habla de distintas realidades del trabajo, haciendo alusión las prácticas, procesos y realidades organizacionales tanto formales como informales.

La relevancia del $\mathrm{CO}$ reside en la capacidad que este fenómeno ha demostrado tener de afectar una amplia gama de fenómenos organizacionales tales como el compromiso (Dávila de León \& Jiménez García, 2014; Noordin, Safiah, Syakirarohan \& Shukriah, 2010), la implementación de procesos de cambio (Pérez de Maldonado \& Maldonado Pérez y Bustamante Uzcategui, 2006), el apoyo que se percibe por parte de la organización (Toro, 2003), la presentación de comportamientos éticos y la disminución de conductas de acoso psicológico (Londoño, M.E., 2009; Muñoz Flores, Guerra De Los Santos, Barón Duque \& Munduate Jaca, 2006), el engagement (Martinez-Lugo, 2009), una menor presentación de factores de riesgo psicosocial (Contreras, Juárez, Barbosa, \& Uribe, 2010), la satisfacción de las personas (Manosalvas Vaca, Manosalvas Vaca \& Quintero,2015), la intención de permanencia (Paula, \& Queiroga, 2015), la creatividad, la calidad y buen servicio a nivel individual y grupal, las relaciones interpersonales (García, Reyes \& Sarmiento, 2006) y el desempeño financiero de la empresa (dos Santos, Coda \& Mazzali, 2010). Esto se debe a que las percepciones influyen los juicios y reacciones emocionales, la motivación (García, Reyes, Sarmiento \& 2006; GonzálezVerde, Jiménez \& Reyes- Roldán, 2015; Mahal, 2009) y, por ende, la conducta orientada a cumplir con las prescripciones establecidas para el cargo que se realiza o desempeño laboral (Toro, 2002) y la productividad de las empresas (Toro \& Sanín, 2013).

Aunque existen múltiples modelos para comprender el $\mathrm{CO}$ e instrumentos para medirlo, los diferentes enfoques plantean la necesidad de explorar las percepciones sobre distintos aspectos de la realidad tales como las relaciones interpersonales, el liderazgo, las recompensas (Echeverri \& Cruz, 2014), los procesos organizacionales (Cardenas, Arciniegas \& Barrera, 2009), los equipos de trabajo, el reconocimiento y el compromiso de las personas con su labor (Mahal, 2009). El presente artículo retomó el modelo de diez variables de $\mathrm{CO}$ planteado por Toro por contar con evidencia empírica y teórica en el contexto colombiano (En: Toro, López, García, Londoño \& Sanín, 2010).

En diversas investigaciones realizadas se ha identificado que la calidad del liderazgo es un determinante del $\mathrm{CO}$, debido a que la manera como se perciben las acciones de los jefes inmediatos logra impactar de manera importante la interpretación de otras realidades del trabajo (Goleman, Boyatzis \& Mckee 2002). Los jefes son la principal fuente de información oficial a la que pueden acceder los colaboradores y son los encargados de la consecución de los recursos necesarios para la realización del trabajo (Toro, 2010). Específicamente se ha identificado que el liderazgo es un modulador de la calidad de las relaciones entre los compañeros de trabajo (Stinglhamber y Vandenberghe, 2003) además de lograr que las personas se alineen con la estrategia organizacional y se genere un sentido de misión de la empresa y del trabajo que se realiza (Bass y Riggio, 2006). Pese a lo anterior se ha encontrado que el liderazgo, como parte del $\mathrm{CO}$, suele ser uno de los aspectos evaluados como menos positivos en las organizaciones latinoamericanas (García, 2004; Londoño, 2010). En esta investigación el rol del jefe es muy relevante en la medida que la metodología planteada requiere que sea quien lidere la elaboración y puesta en marcha del plan de intervención (Toro \& Sanín, 2013).

Los procesos de intervención del $\mathrm{CO}$ que se planteen en las organizaciones deben estructurarse como acciones planeadas, basadas en la teoría, que busquen mejorar la salud y el bienestar de los empleados a través del cambio de la forma en que el trabajo es diseñado, organizado y gestionado (LaMontagne et al., 2007; Nielsen, 2013). 
El proceso de intervención planteado en este artículo siguió los pasos propuestos por Toro y Sanín (2013) quienes plantean un proceso de gestión participativo, en el que los colaboradores dejan de ser simples receptores de los procesos diseñados por la organización o por gestión humana (Nielsen, 2013). El trabajo participativo es bastante común en los procesos de intervención que se encuentran en la literatura (Cardenas, Arciniegas \& Barrera, 2009; García, Reyes \& Sarmiento, 2006; MacDonald, 2013).

La metodología mencionada incluye, en primer lugar, el análisis o medición objetiva del CO. En esta fase se hace necesario la utilización de instrumentos válidos y confiables dado que suministra la información en la que se basará todo el proceso de intervención. Se debe verificar que la muestra de participantes sea representativa de la población total de la organización. No obstante, idealmente se debería invitar a todos los colaboradores dado que esto favorecerá el compromiso en las siguientes fases (Nielsen \& Randall, 2012).

En segundo lugar, la explicación o socialización de los resultados a los miembros de los equipos de trabajo. Este paso es necesario para que las personas conozcan la relevancia del tema, comprendan los resultados y empiecen a comprometerse con la gestión del mismo.

Posteriormente, teniendo en cuenta que los diagnósticos de $\mathrm{CO}$ suelen ser descriptivos más no explicativos, se debe realizar una profundización con el fin de identificar las condiciones que justifican los resultados obtenidos partiendo de la visión de que son los colaboradores quienes mejor conocen las realidades que enfrentan en su día a día laboral. Para cumplir este objetivo se pueden emplear diversos métodos grupales tales como los grupos focales, entrevistas grupales, grupos nominales o entrevistas individuales dependiendo del tamaño y los resultados obtenidos. En esta etapa podrá ser muy beneficioso plantear un método que favorezca la participación confidencial de las personas con el fin de garantizar su sinceridad y participación (García, Reyes \& Sarmiento, 2006). Como resultado de esta fase se tendrá un listado de hechos cotidianos que explican los resultados de CO.

La búsqueda de las explicaciones es muy importante dado que, con base en estas, el grupo debe plantear un plan de mejoramiento el cual debe incluir preferentemente acciones que estén bajo la injerencia de los integrantes del grupo, definir claramente los responsables de la implementación y la definición de indicadores de logro que permitan identificar el cambio en los aspectos registrados como oportunidades de mejora. Esto permitirá el planteamiento de una estrategia ajustada a las realidades de cada organización (García, Reyes \& Sarmiento, 2006). Esta metodología sugiere que, en caso de que existan varias oportunidades de mejora en los resultados de los grupos, en esta fase se priorice el planteamiento de acciones para la mejora de las variables que han demostrado tener un mayor impacto en la calidad general del $\mathrm{CO}$, estas son Apoyo del Jefe, Trato Interpersonal, Claridad Organizacional y Disponibilidad de Recursos (Londoño, 2010; Toro, 2008; Toro, 2010b; Toro \& Sanín, 2013).

A continuación, los grupos deben poner en marcha la intervención diseñada durante al menos tres meses con el fin de que se afiancen las nuevas prácticas y empiecen a darse cambios en las percepciones de los colaboradores. Por último, se verifica la eficacia de las acciones planteadas, puede ser mediante un nuevo estudio de la calidad del $\mathrm{CO}$, pero también con la revisión de los indicadores de logro propuestos en los planes de gestión.

\section{Método}

El estudio es de tipo cuantitativo, preexperimental con un diseño pre-prueba/pos prueba con un solo grupo (Hernández, Fernández \& Baptista, 2010; Kerlinger \& Lee, 2002). Se tenía como hipótesis que la intervención diseñada 
y ejecutada produciría cambios significativos en la calidad del clima organizacional.

\section{Muestra}

En la primera medición participaron 157 colaboradores. En la segunda participaron 169 colaboradores. En ambos diagnósticos se invitó al tota de la población de la empresa sin embargo hay diferencia en el número total debido a algunos cambios de personal dentro de la organización y a la disponibilidad de quienes quisieron hacer parte del estudio. Todos los participantes contaban con vinculación laboral directa con la empresa. Dada la diferencia en la muestra, y buscando guardar la confidencialidad de la encuesta, los datos se emparejaron por áreas lo que permitió comparar doce grupos distintos, antes y después de la intervención, es decir, no se compararon respuestas individuales en el pre y el pos, sino que se compararon perfiles por grupos. El 5\% de los encuestados tenía entre 18 y 30 años, el 57\% tenía de 31 a 50 años y el porcentaje restante era mayor de 50 años. Se contó con participación de personal de distintos niveles educativos. El 10\% tenía básica primaria, el $20 \%$ eran bachilleres, el $15 \%$ poseía formación técnica o tecnológica, el $25 \%$ eran profesionales y el resto tenía estudios de posgrado. El $24 \%$ de los participantes tenía cargos de jefatura.

\section{Procedimiento}

El proyecto fue revisado $\mathrm{y}$ avalado por el comité científico de CINCEL S.A.S. quien consideró aspectos éticos, teóricos $\mathrm{y}$ metodológicos que hicieran viable su desarrollo. Se solicitó el consentimiento informado a todos los participantes. El $66 \%$ de los encuestados respondieron los instrumentos de forma virtual a través de un aplicativo que llegaba al correo electrónico, el $34 \%$ restante la diligenció en formato de lápiz y papel. Las encuestas presenciales estuvieron a cargo de profesionales externos capacitados para ello y se aplicaron en grupos de aproximadamente 25 personas. Las encuestas virtuales se capturaban a través de la plataforma de surveymonkey. Las presenciales se leyeron haciendo uso del Teleform (10.8). Luego de tener consolidadas las encuestas se pasaron a SPSS.21, software en el cual se organizó y analizó la información.

La recolección de información correspondiente al pre-test se realizó en julio de 2013. Antes de iniciar propiamente la gestión se llevó a cabo una reunión con el gerente general y varios de los directivos para presentar los resultados y verificar su respaldo al proceso, en esta parte se aclara que el rol del consultor es de experto que acompaña el proceso, que la intervención propiamente es responsabilidad de los equipos y de la dirección. La intervención se llevó a cabo entre marzo y agosto de 2014 y comprendió cuatro etapas. La primera fue un taller de ocho horas dirigido a todos los líderes de la organización cuyo objetivo era prepararlos para gestionar en los grupos de la organización la correcta comprensión de los resultados del diagnóstico del clima organizacional, la promoción de su análisis y la elaboración colectiva de planes de mejoramiento.

La segunda etapa consistió en el acompañamiento en la identificación de realidades particulares con cada uno de los grupos y en la generación de propuestas de acción por parte de los colaboradores. Para ello se utilizó la metodología basada en los grupos nominales (Van de Ven $\mathrm{AH}$, Delbecq AL, 1972) donde se reunían entre 6 y 12 personas de un mismo equipo quienes escogían dos variables sobre las cuales querían focalizar la gestión. Los participantes tenían la posibilidad de escribir de forma anónima todas las razones por las cuales consideraban que dicho aspecto del CO podía estar siendo afectado. Posteriormente, las explicaciones eran leídas por el facilitador y cada participante calificaba su nivel de acuerdo con cada una de las frases. Al terminar el ejercicio se contaba con un listado de explicaciones y con el nivel de acuerdo del grupo frente a ellas. Con 
esta información se subdividía el grupo y se les daban algunas indicaciones para que iniciaran la búsqueda de soluciones. Una vez finalizada la actividad se realizaba una reunión entre el consultor y el jefe del equipo para retroalimentar a este último sobre lo encontrado. En esta segunda fase se trabajó con un total de 15 grupos. Las variables más trabajadas fueron apoyo del jefe y claridad organizacional (en diez de los grupos), seguidas de trato interpersonal (en cinco grupos). Los grupos restantes trabajaron aspectos como disponibilidad de recursos, coherencia, trabajo en equipo y retribución.

La tercera fase correspondió a la realización e implementación de los planes de mejoramiento. Cada grupo debía reunirse con su líder para diseñar las actividades, formular indicadores de seguimiento y de logro y asignar responsables de ejecución y de verificación. Una vez el plan estuvo diseñado fue revisado por el consultor quien sugirió algunos ajustes a fin de que las acciones fueran viables y tuvieran impacto sobre las variables elegidas.

La cuarta etapa consistió en el seguimiento y ajuste de los planes. Para ello el consultor se reunió nuevamente con cada uno de los grupos y de los jefes para revisar la ejecución de las actividades, las dificultades encontradas y el cumplimiento de los indicadores planteados.

La segunda medición se hizo en julio de 2015 y a partir de allí se realizaron los análisis correspondientes.

\section{Análisis de datos}

Después de calcular los datos descriptivos, se verificó con Kolmogorov-Smirnov la distribución de los datos. Dado que estos se distribuyeron de forma paramétrica $(\mathrm{p}>0.005)$ se utilizó la prueba $t$ de muestras relacionadas para determinar si las diferencias entre el pre-test y el pos-test eran significativas. Finalmente se calculó el tamaño del efecto con la $d$ de Cohen.

\section{Instrumentos}

Se utilizó la Encuesta de Clima Organizacional ECO - Versión IV la cual fue validada con 35.762 casos correspondientes a 56 empresas y 10 países. Esta prueba tiene 64 ítems que se agrupan en diez variables y a los que se debe responder con una escala tipo Likert de 4 puntos, donde 1 es completamente en desacuerdo y 4 completamente de acuerdo. Este instrumento tiene una confiabilidad de 0.93 calculada con el método Alpha de Cronbach y validez constatada mediante análisis factorial con el método de extracción de ejes principales y rotación promax. La varianza total explicada con las diez dimensiones es del $58.6 \%$.

En la Tabla 1 se enuncian las variables y la definición de cada una de ellas.

\section{Tabla 1}

Definición de las variables exploradas con la Encuesta de Clima Organización ECO IV

\begin{tabular}{|c|c|}
\hline Variable & Definición \\
\hline Apoyo del Jefe & $\begin{array}{l}\text { Percepción del grado en que el jefe } \\
\text { respalda, estimula y da participación } \\
\text { a sus colaboradores. }\end{array}$ \\
\hline $\begin{array}{c}\text { Claridad } \\
\text { Organizacional }\end{array}$ & $\begin{array}{l}\text { Grado en que el personal percibe que } \\
\text { ha recibido información apropiada } \\
\text { sobre su trabajo y el funcionamiento } \\
\text { de la organización. }\end{array}$ \\
\hline Trato Interpersonal & $\begin{array}{l}\text { Percepción del grado en que el } \\
\text { personal se ayuda entre sí y sus } \\
\text { relaciones son de respeto. }\end{array}$ \\
\hline $\begin{array}{l}\text { Disponibilidad de } \\
\text { Recursos }\end{array}$ & $\begin{array}{l}\text { Percepción del grado en que el } \\
\text { personal cuenta con los equipos, los } \\
\text { implementos y el aporte requerido } \\
\text { de otros para la realización de su } \\
\text { trabajo. }\end{array}$ \\
\hline $\begin{array}{c}\text { Apoyo } \\
\text { Organizacional }\end{array}$ & $\begin{array}{l}\text { Creencia acerca del grado de } \\
\text { atención personal, interés y apoyo } \\
\text { que el empleado obtiene de la } \\
\text { organización. }\end{array}$ \\
\hline Estabilidad & $\begin{array}{l}\text { Grado en que los empleados ven } \\
\text { claras posibilidades de permanencia } \\
\text { de la organización y estiman que a } \\
\text { la gente se la conserva o despide con } \\
\text { criterio justo. }\end{array}$ \\
\hline
\end{tabular}


Tabla 1

Definición de las variables exploradas con la Encuesta de Clima Organización ECO IV (continuación)

Variable

Definición

\begin{tabular}{|c|c|}
\hline Retribución & $\begin{array}{l}\text { Grado de equidad percibida en } \\
\text { la remuneración y los beneficios } \\
\text { derivados del trabajo. }\end{array}$ \\
\hline Coherencia & $\begin{array}{l}\text { Percepción de la medida en que } \\
\text { las actuaciones del personal y de } \\
\text { la organización se ajustan a los } \\
\text { principios, objetivos, normas y } \\
\text { reglamentos establecidos. }\end{array}$ \\
\hline $\begin{array}{l}\text { Sentido de } \\
\text { Pertenencia }\end{array}$ & $\begin{array}{l}\text { Percepción del grado de orgullo, } \\
\text { compromiso y responsabilidad } \\
\text { derivado de la vinculación a la } \\
\text { organización. }\end{array}$ \\
\hline Trabajo en Equipo & $\begin{array}{l}\text { Grado en que se percibe la } \\
\text { existencia de un modo organizado } \\
\text { de trabajar en equipo y que este es } \\
\text { conveniente para el empleado y para } \\
\text { la organización. }\end{array}$ \\
\hline
\end{tabular}

\section{Resultados}

La prueba Kolmogorov-Smirnov arrojó niveles de significancia superiores a 0,05 para todas las variables tanto en el pre-test como en el pos-test indicando que los datos se distribuían de forma normal y que se debía hacer uso de una prueba paramétrica para la comparación de las mediciones.

La Tabla 2 muestra los datos descriptivos de las variables, los resultados de la prueba $\mathrm{t}$ para muestras relacionadas con su respectivo nivel de significancia y el tamaño del efecto en cada comparación. A excepción de retribución todas las demás variables obtuvieron una mejora significativa $(p<0,05)$ entre el pre-test y el postest. El tamaño del efecto se considera grande para todas las comparaciones (Coe \& Merino, 2003) siendo el cambio más importante el de estabilidad y el más bajo el de trato interpersonal.

Tabla 2

Comparación de los resultados del primer y segundo estudios

\begin{tabular}{|c|c|c|c|c|c|c|}
\hline Variable & $\begin{array}{c}\text { Año de } \\
\text { medición }\end{array}$ & Media & $\begin{array}{c}\text { Desviación } \\
\text { estándar }\end{array}$ & Prueba T & $\begin{array}{c}\text { Sig. } \\
\text { (bilateral) }\end{array}$ & $d$ de Cohen \\
\hline \multirow{2}{*}{ Apoyo del jefe } & 2013 & 16,35 & 1,52 & $-3,144$ & ,009 & 1,13 \\
\hline & 2015 & 18,47 & 2,24 & & & \\
\hline \multirow{2}{*}{ Claridad organizacional } & 2013 & 17,75 & 1,34 & $-3,242$ & ,008 & 1,21 \\
\hline & 2015 & 19,20 & 1,06 & & & \\
\hline \multirow{2}{*}{ Trato interpersonal } & 2013 & 18,60 & 1,64 & $-2,850$ &, 016 & 0,83 \\
\hline & 2015 & 19,88 & 1,46 & & & \\
\hline \multirow{2}{*}{$\begin{array}{l}\text { Disponibilidad de } \\
\text { recursos }\end{array}$} & 2013 & 17,90 & 1,51 & $-3,047$ & 011 & 1,11 \\
\hline & 2015 & 19,60 & 1,54 & & & \\
\hline \multirow{2}{*}{ Estabilidad } & 2013 & 20,15 & 0,99 & $-5,464$ &, 000 & 1,61 \\
\hline & 2015 & 21,72 & 0,96 & & & \\
\hline \multirow{2}{*}{ Retribución } & 2013 & 21,32 & 1,39 & $-1,223$ & 247 & - \\
\hline & 2015 & 21,67 & 1,28 & & & \\
\hline \multirow{2}{*}{ Coherencia } & 2013 & 19,59 & 1,18 & $-2,788$ &, 018 & 1,20 \\
\hline & 2015 & 20,82 & 0,88 & & & \\
\hline \multirow{2}{*}{ Sentido de Pertenencia } & 2013 & 19,53 & 1,27 & $-3,093$ &, 010 & 1,11 \\
\hline & 2015 & 20,70 & 0,82 & & & \\
\hline \multirow{2}{*}{ Trabajo en Equipo } & 2013 & 17,73 & 1,87 & $-4,121$ &, 002 & 1,30 \\
\hline & 2015 & 19,84 & 1,37 & & & \\
\hline \multirow{2}{*}{ Apoyo Organizacional } & 2013 & 16,16 & 1,82 & $-2,760$ & ,019 & 0,95 \\
\hline & 2015 & 18,07 & 2,20 & & & \\
\hline
\end{tabular}




\section{Discusión}

El objetivo del estudio era verificar el efecto de la intervención del $\mathrm{CO}$ en una organización específica. Los hallazgos del estudio mostraron que tras la intervención sistemática se obtuvieron cambios significativos en la calidad del clima organizacional en los grupos. Es importante resaltar que para el logro de estos resultados fueron esenciales la participación y el empoderamiento de los colaboradores para la generación de espacios de trabajo más saludables (Aust y Ducki, 2004; OMS, 2010). La participación parte de la premisa de que las personas son quienes mejor conocen sus condiciones de trabajo y que esta experticia puede usarse en el desarrollo de sugerencias para mejorar la situación (Brandenburg y Slesina, 1994) generando un mayor compromiso para la ejecución de las acciones propuestas (OMS, 2010). En este escenario entonces el papel de las áreas de Gestión Humana es prestar el apoyo necesario a los equipos para plantear sus planes y hacer seguimiento al cumplimiento de los mismos.

Otra condición que resultó clave para lograr los objetivos de la intervención fue el trabajo con los jefes del equipo el cual buscaba generar un mayor compromiso por parte de ellos para el mejoramiento del clima y desarrollar habilidades para que pudieran asumir su rol de manera más exitosa. En general el fortalecimiento de la calidad del liderazgo redundará en percepciones más convenientes sobre otros aspectos del trabajo (Toro, 2010b) dado que los jefes personifican, integran y cambian aspectos de la cultura que finalmente impactan el CO (Molero, 2002).

El respaldo y compromiso de la dirección también fue uno de los determinantes de la gestión del $\mathrm{CO}$ dado que desde allí se realiza el requerimiento de cambio y se asignan recursos (Toro, Sanín, \& 2012). Es el nivel gerencial y directivo de una organización el que decide qué medir y controlar, el que orienta el manejo de las crisis a nivel empresarial, y el que define las políticas que guían en gran parte las actuaciones del personal.
A pesar de que los distintos planes de acción se enfocaban en solo dos variables, los análisis muestran que, a excepción de una de ellas, todas las demás mostraron mejoras significativas. Ello se explica por el carácter dinámico del $\mathrm{CO}$ en el que los distintos atributos interactúan y se influyen entre sí. Además, si bien los participantes de los grupos eran libres de escoger aquellos aspectos sobre los cuales se focalizaría el plan de acción, el consultor procuraba que la gestión se centrara en las variables estratégicas, es decir, en aquellas que habían demostrado mayor capacidad de predicción de la calidad global del CO (Toro \& Sanín, 2013). De allí, que las variables más trabajadas fuesen apoyo del jefe, claridad organizacional y trato interpersonal.

Trato Interpersonal es la variable que presentó menor variación. Pese a que muchos de los planes de intervención se enfocaron en este aspecto, es importante tener en cuenta que la percepción es más resistente al cambio cuando se trata de fenómenos sociales (Beck, 1979). La naturaleza social del ser humano conlleva a que la interacción con los otros determine la forma en que se interpreta el mundo y se interactúa con él (Lera, 2016) por lo que dicho cambio, aun siendo el menor, se considera de gran importancia.

La única variable cuyas puntuaciones fueron estables es Retribución. Dicho aspecto cuando no es bien percibido, suele tener una incidencia negativa sobre la capacidad de la organización para atraer y retener personal valioso (Toro \& Sanín, 2013). Una percepción favorable de la equidad en la retribución puede impactar positivamente los índices de rotación y ausentismo, y disminuir los costos de operación para la organización (Toro, 2002). Sin embargo, era esperable que la gestión no impactara dicha condición pues los cambios en esta variable suelen obedecer más a modificaciones en políticas administrativas que a las gestiones que pueda realizar el personal. De forma contraria, Estabilidad es la variable con mayor incremento en la puntuación luego de la intervención. No 
obstante, dicho cambio puede deberse también a decisiones administrativas relacionadas con la vinculación de personas que pasaron de ser contratistas a trabajar directamente con la organización.

\section{Limitaciones}

Pese a que se logró evaluar el efecto positivo de la metodología de intervención, el estudio presenta algunas limitaciones. Una de ellas corresponde al diseño en tanto que al carecer de grupo de control y no hacer manipulación o control de otras variables no se puede asegurar plenamente que los cambios sean producto de la intervención. Además, por ser el estudio de un caso no es posible afirmar que en organizaciones de otro sector o con algunas características particulares se obtengan los mismos resultados.

Durante la elaboración de los planes de acción los colaboradores manifestaron inconformidad debido a que las personas que prestaban algún tipo de servicio dentro de la organización pero que no tenían contrato directo con la misma, no participaron del proceso. El $\mathrm{CO}$ es un fenómeno que trasciende la estructura formal de la organización y que impacta a todos aquellos que comparten un mismo entorno independientemente de lo que formalmente establezca el contrato, por ello, aunque es una deficiencia que estas personas no hayan asistido directamente a los espacios de divulgación de resultados y de construcción de planes, es probable que se hayan visto impactados de forma positiva por las acciones implementadas.

Por razones administrativas se realizó un solo seguimiento por lo que en este momento se desconoce la estabilidad o mejora de los resultados. En relación con este aspecto, se ha encontrado que es necesario que los procesos de mejoramiento de las condiciones laborales sigan un ciclo de mejoramiento continuo (OMS, 2010), revisando los logros alcanzados con las acciones propuestas con el fin de enfocarse en nuevos aspectos una vez se han cumplido los objetivos planteados. También se sugiere ampliar el proceso de formación a los líderes para asegurar que se desarrollen las competencias requeridas para seguir acompañando el proceso de gestión.

Se concluye que una gestión sistemática y participativa del CO logra cambios importantes que favorecen la calidad de vida de las personas y la productividad organizacional.

\section{Referencias}

Argyris, C. (1957). Personality and organization. New York: Harper and Row

Aust, B. y Ducki, A. (2004) Comprehensive Health Promotion Interventions at the Workplace: Experiences With Health Circles. Germany Journal of Occupational Health Psychology. 9(3), 258-270 10768998/04/\$12.00 DOI: 10.1037/10768998.9.3.258

Bass, B. y Riggio, R. (2006) Transformational Leadership. Second Edition. Lawrence Erlbaum Associates Publishers, Mahwah.

Beck, A. T. (Ed.). (1979). Cognitive therapy of depression. Guilford press.

Brandenburg, U., \& Slesina, W. (1994). Health promotion circles: A new approach to health promotion at worksite. Homeostasis in Health and Disease, 35(1-2), 43-48.

Brunet, L. (1987). El clima de trabajo en las organizaciones: Definición, Diagnóstico y Consecuencias Editorial Trillas. México.

Cardenas, 1. Arciniegas, J., Barrera, M. (2009) Modelo de Intervención de Clima Organizacional. International Journal of Psychological research. 2 (2). 121-127 
Chiavenato, I. (1992). Introducción a la Teoría General de la Administración. Tercera Edición. Editorial Mc Graw Hill. México. D.

Coe, R. \& Merino, C. (2003). Magnitud del Efecto: Una guía para investigadores y usuarios. Revista de Psicología de la PUCP, 21 (1), 147-177.

Contreras, F., Juárez, F., Barbosa, D. \& Uribe, A.F. (2010). Estilos de Liderazgo, Riesgo Psicosocial y Clima Organizacional en un Grupo de Empresas Colombianas. Revista Facultad Ciencias Económicas. XVIII (2), 7-17.

Dávila de León, C. \& Jiménez García, G. (2014). Sentido de pertenencia y compromiso organizacional: predicción del bienestar. Revista de Psicología, 32(2), 272-302.

Davis, K. (1991). Comportamiento Humano en el Trabajo. Editorial Mc Graw Hill. México.

dosSantos, M.,Coda, R., Mazzali,L.(2010)Revista Universo Contábil. Clima Organizacional e Desempenho Financeiro. 6 (2), 27-46.

Echeverri, D. R. C., \& Cruz, R. Z. (2014). Revisión de instrumentos de evaluación de clima organizacional. Estudios Gerenciales, 30 (131), 184-189.

Garcia, A. (2004). Consideraciones para el diagnóstico e intervención de la variable de clima organizacional "Apoyo del jefe". Revista Interamericana de Psicología Ocupacional, 23 (2), 90-93.

García, L., Reyes, A. \& Sarmiento, P. (2006) Clima Organizacional de la Escuela de Odontología de la Universidad del Valle. Revista Estomatología. 14(1), 32-35.

Goleman, D. Boyatzis, R. \& Mckee, A. (2002) Primal Leadership. Realizing the Power of Emotional Intelligence. Boston: Harvard
Gonzalez-Roma, V., Peiró, J.M. \& Tordera, N. (2002) An Examination of the Antecedents and Moderator Influences of Climate Strength. Journal of Applied Psychology; 87 (3), 465-473.

González-Verde, A., Jiménez, A. \& ReyesRoldán, M. (2015). Procedimiento para el Diagnóstico y Mejora del Clima Organizacional. Revista Ingeniería Industrial. 14 (2), 25-42.

La MontagneAD, Keegel TG. Whatorganizational/ employer level interventions are effective for preventing and treating occupational stress? A Rapid review for the Institute for Safety, Compensation and Recovery Research. Disponible en: http://iscrr. com.au/media/38371/employer\%20 interventions $\% 20$ for $\% 20$ preventing $\% 20$ stress.pdf.

Lera, M. (2016). La naturaleza social del ser humano. In M. Lera, Paradigmas en educación y desarrollo (1st ed.). Retrieved from http://www.psicoeducacion.eu/ eduinfantil/Cap_IV._La_naturaleza social_del_ser_humano.pdf

Londoño, M.E. (2009) Relaciones entre Clima Organizacional, Acoso Psicológico y los Comportamientos Éticos en el trabajo. Estudio de un caso. Revista Interamericana de Psicología Ocupacional. 28(2)

Londoño, M.E. (2010). Diferencias en Apoyo del Jefe e Imagen Gerencial en Muestras de Colombia, Costa Rica, Ecuador, Panamá, Perú y Venezuela. En Clima Organizacional, Una aproximación a su dinámica en la empresa latinoamericana. Segunda Edición Medellín: Cincel.

Mahal, P. (2009) Organizational Culture and Organizational Climate as a Determinant of Motivation The IUP Journal of Management Research. 8 (10), 38-51. 
Manosalvas Vaca, C., Manosalvas Vaca, L., Quintero, J. (2015) El Clima Organizacional y la Satisfacción Laboral: Un análisis cuantitativo riguroso de su relación. Ad-Minister. 26, 5-15.

Martinez-Lugo, M. (2009). La vinculación psicológica en el trabajo (engagement), el síndrome de quemarse por el trabajo y el clima organizacional en un grupo de empleados en Puerto Rico. Revista Interamericana de Psicología Ocupacional 28 (1) 7 -15.

MacDonald, P. (2013). Organizational climate assessment and improvement planning Chapter 4. Workplace Culture in Academic Libraries. The early 21 Century. Blessinger, K \& Hrycaj, P. (Ed) Chandos Publisinhy

Méndez Álvarez, C. (2006). Clima organizacional en Colombia, Bogotá. El IMCOC: Un método de análisis para su intervención. Centro Editorial Universidad del Rosario.

Molero, F. (2002). Cltura y Liderazgo una Relación Multifacética. Boletín De Psicología, (76), 53-75. Retrieved from http://www.uv.es/ seoane/boletin/previos/N76-4.pdf

Nielsen, K. (2013). Review Article: How can we make organizational interventions work? Employees and line managers as actively crafting interventions. Human Relations, 66(8), 1029-1050.

Nielsen, K. y Randall, R. (2012) The importance of employee participation and perceptions of changes in procedures in a teamworking intervention, Work \& Stress, 26 (2), 91-111. DOI: $10.1080 / 02678373.2012 .682721$

Noordin, F., Safiah, O., Syakirarohan, Sy Shukriah, I. (2010). Organizational Climate And Its Influence On Organizational Commitment. The International Business \& Economics Research Journal, 9(2). 1-9.
Organización Mundial de la Salud(2010). Entornos Laborales Saludables: Fundamentos y Modelo de la OMS- Contextualización, prácticas y Literatura de Apoyo [Internet]. [Consultado 2012 may 15]. Disponible en: http://www.who.int/occupational_health/ evelyn_hwp_spanish.pdf

Ostroff, C., Kinicki, A., \& Tamkins, M. (2003) Chapter 22. Organizational Culture and Climate. En: Handbook of Psychology Volume 12. Industrial and Organizational Psychology. Borman, W., Ilgen, D., Klimoski,R. Volume Editors Irving B. Weiner Editor-in-Chief. John Wiley \& Sons, Inc., Hoboken.

Paula, Alexandre Pinto Vieira de, \& Queiroga, Fabiana. (2015). Satisfação no trabalho e clima organizacional: a relação com autoavaliações de desempenho. Revista Psicologia Organizações e Trabalho, 15(4), 362-373. DOI: 10.17652/ $\operatorname{rpot} / 2015.4 .478$

Perez de Maldonado, I. Mldonado Pérez, Marisabel y Bustamante Uzcategui, S. (2006) Clima organizacional y gerencia: inductores del cambio organizacional. Investigación y Postgrado [online]. 21 (2) 231-248. Disponible en: $\quad<$ http://www.scielo.org.ve/scielo. php? script $=$ sci_arttext\&pid $=\mathrm{S} 1316$ 00872006000200009\&lng $=$ es \& nrm=i so>. ISSN 1316-0087.

Reichers, A. E. \& Schneider, B. (1990). Climate and Culture: An evolution of constructs. En Schneider, B. (Ed.).Organizational Climate and Culture. San Francisco: Jossey-Bass.

Stinglhamber, F. \& Vandenberghe, C. (2003). Organizations and supervisors as sources of support and targets of commitment: a longitudinal study. Journal of Organizational Behavior, 24, 251-270. 
Toro, F. \& Cabrera, C. (1998) Distinciones y relaciones entre clima, motivación, satisfacción y cultura organizacional. Revista Interamericana de Psicología Ocupacional, 17 (2), 27-39.

Toro, F., López, R., García, A., Londoño M. \& Sanin A. (2010) Clima Organizacional, una aproximación a su dinámica en la empresa Latinoamérica. Segunda Edición. Medellín: Cincel.

Toro, A. \& Sanin, A.(Ed) (2013). Gestión del Clima Organizacional: Intervención basada en evidencias. Cincel: Medellín.

Toro, F. (2002) Bases conceptuales para el diseño de un instrumento de diagnóstico de la satisfacción laboral en Colombia. Revista Interamericana de Psicología Organizacional, 21(2), 18-25.

Toro. F. (2002). Desempeño y Productividad. Medellín: Cincel.

Toro, F. (2003) Incidencia del clima organizacional sobre el apoyo organizacional percibido. Revista Interamericana de Psicología Ocupacional. 22 (2), 77-83.
Toro, F. (2008) Relación entre los Estilos de Influencia de los jefes y el Clima Organizacional. Revista Interamericana de Psicología Ocupacional. 27 (2), 133145.

Toro, F. (2010) Efectos sobre el clima organizacional de una experiencia de formación de los líderes. En: Toro, F. Clima Organizacional. Una aproximación a su dinámica en la empresa latinoamericana (segunda edición) (pp. 291-298). Medellín: Cincel.

Toro, F (Ed.) (2010b). Clima Organizacional, Una aproximación a su dinámica en la empresa latinoamericana. Segunda Edición Medellín: Cincel.

Van de Ven AH, Delbecq AL.(1972) The Nominal group as a research instrument for exploratory health studies. American Journal of Public Health. 62. 337-342.

Vega, D., Arévalo, A., Sandoval, J., Aguilar Bustamante, M., \& Giraldo, J. (2006). Panorama sobre los estudios de clima organizacional en Bogotá, Colombia (1994-2005). Divers.: Perspect. Psicol., 2(2), DOI: 10.15332/s17949998.2006.0002.12 